\title{
Enantioselective Conjugate Addition of Fluo romalonate to Nitroalkenes Catalyzed by Chiral Nickel Complexes
}

\author{
Seung Hee Kang and Dae Young Kim \\ Department of Chemistry, Soonchunhang Linersity, Asan, Chungnam 336-745, Korea. *E-mail: droungáschackr \\ Received April 28, 2009. Accepted Mav 22, 2009
}

Key Words: Chiral nickel complexes. Michael reaction. Nitroalkenes. Așymmetric catalysis. Fluoromalonates

Fluorinated compounds are of importance in organic synthesis because of their use as medicines and agrochemicals and in fundamental studies of biochemical and metabolic processes. ${ }^{\text {' }}$ Introduction of fluorine atom into biologically active compounds often leads to improvement of their biological characteristics due to unique properties of the fluorine atom. ${ }^{2}$ Chiral organofluorine compounds have been utilized in studies of enzyme mechanisms and as intermediates in asymmetric syrntheses. ?

The Michael addition reaction is widely recognized as one of the most general and versatile methods for formation of $\mathrm{C}-\mathrm{C}$ bonds in organic synthesis, ${ }^{4}$ and the development of enantioselective catalytic protocols for this reaction has been subject of intensive research. ${ }^{5}$ Michael reaction of nucleophiles to nitroalkenes represents a direct and most appealing approach to chiral nitroalkanes that are versatile intermediates in organic synthesis. which can be transformed into an amine, nitrile oxide. ketone. carboxylic acid. and hydrogen etc.

Recently. several groups presented the cataly tic asymmetric conjugate additions of active methine compounds to nitroalkenes in the presence of chiral metal complexes or organocatalysts. ${ }^{8}$ Although catalytic enantioselective Michael additions of 1.3-dicarbonyl compounds have reported. up to now there is one example of these reactions with fluoromalonates using chiral Mg-box complex. ${ }^{9 b}$ However. a highly enantioselective conjugate addtion of fluoromalonates to nitroalkenes remains elusive: although. if successfully promoted with a practically accessible chiral catalyst under air- and moisture-tolerant conditions. it could provide a highly attractive. convergent approach toward optically active $\gamma$-nitro fluoromalonates.

As part of research program related to the development of synthetic methods for the enantioselective construction of stereogenic carbon centers. "we recently reported chiral nickel complexes 1 (Fig. 1) to be a highly selective cataly st for the enantioselective amination of active methines. "In this communications. we wish to describe the direct asymmetric Michael reaction of fluoromalonates to nitroalkenes catalyzed

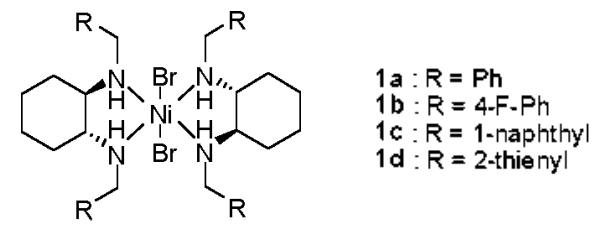

Figure 1. Structure of chiral nickel complexes. by air- and moisture-stable chiral nickel complexes. ${ }^{12}$

A survey of some reaction parameters was performed and some representative results are presented in Table 1. Our investigation began with the catalytic asymmetric Michael addition of ethyl fluoromalonate (2) with $\beta$-nitrosty rene (3a). When the reaction was performed in toluene at room temperature in the presence of $5 \mathrm{~mol} \%$ catalyst 1a. product ta was isolated in high yield with $97 \%$ ee (Table 1 . entry 1 ). We first examined the impact of the structure of catalysts 1a-d on enantioselectivities (Table $1,87-97 \%$ ee, entries $1-4$ ). The best results have been obtained with catalyst 1a Concerning the solvents (entries I and 5-1 l). the use of nompolar solvents gave the good results in the yield and the enantiomeric excess.

To examine the generality of the catalytic asymmetric Michael reaction of fluoromalonates 2 by using chiral nickel catalyst 1a. we studied the addition of fluoromalonates 2 to wide range of substituted aromatic and heteroaromatic nitroalkenes 3 in toluene. ${ }^{13}$ As it can be seen by the results summarized in Table 2. the corresponding products ta-h were obtained in excellent yields ( $89-97 \%$ ) and excellent enantioselectivities (90-97\% ee). The absolute configuration was determined after transesterification ${ }^{15}$ of + to corresponding dimethỵl malonate derivatives by comparing chiral HPLC

Table 1. Optimization of the reaction conditions

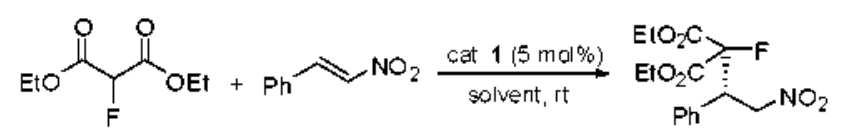

\begin{tabular}{|c|c|c|c|c|c|}
\hline entry & cat. 1 & solvent & time (h) & yield $(\%)$ & $2 e^{b}(\%)$ \\
\hline 1 & $1 \mathrm{a}$ & toluene & 20 & 97 & 97 \\
\hline 2 & 1b & toluene & 20 & 93 & 95 \\
\hline 3 & $1 \mathrm{c}$ & toluene & 20 & 91 & 87 \\
\hline 4 & 1d & toluene & 20 & 90 & 91 \\
\hline 5 & $1 \mathfrak{a}$ & $\mathrm{CH}_{2} \mathrm{Cl}_{2}$ & 15 & 90 & 93 \\
\hline 6 & la & o-xylene & 20 & 95 & 97 \\
\hline 7 & la & $m$-xylene & 20 & 95 & 96 \\
\hline 8 & la & $p$-xylene & 20 & 96 & 95 \\
\hline 9 & la & EtOAc & 24 & 85 & 95 \\
\hline 10 & $1 a$ & $\mathrm{EtOH}$ & 12 & 96 & 91 \\
\hline 11 & la & $\mathrm{THF}$ & 16 & 91 & 92 \\
\hline
\end{tabular}

Isolated yields. ${ }^{b}$ Enantiopurity of ta was determined by HPLC analysis with a Chiralpak AD-H column. 
Table 2. Enantioselective conjugate addition of fluoromalonate to nitroalkenes

\begin{tabular}{|c|c|c|c|c|}
\hline & & & & 4 \\
\hline entry & 3, AT & time (h) & yield $(\%)$ & $e^{b}(\%)$ \\
\hline l & $\mathrm{C}_{n} \mathrm{H}_{\xi}$ & 20 & $4 a, 97$ & 97 \\
\hline 2 & $4-\mathrm{F}-\mathrm{C}_{6} \mathrm{H}_{4}$ & 11 & $4 b, 92$ & 93 \\
\hline 3 & $4-\mathrm{OMe}-\mathrm{C}_{5} \mathrm{H}_{4}$ & 10 & $4 c, 91$ & 95 \\
\hline 4 & $4-\mathrm{Me}-\mathrm{C}_{n} \mathrm{H}_{4}$ & 9 & $4 d, 95$ & 91 \\
\hline 5 & $2-\mathrm{F}-\mathrm{C}_{6} \mathrm{H}_{4}$ & 9 & $4 \mathrm{e}, 92$ & 91 \\
\hline 6 & $2-\mathrm{Cl}_{-}-\mathrm{C}_{6} \mathrm{H}_{4}$ & 13 & $4 f, 96$ & 97 \\
\hline 7 & 2-furyl & 19 & $4 g, 89$ & 90 \\
\hline 8 & 2-thienyl & 10 & th. 90 & 91 \\
\hline
\end{tabular}

"Isolated yields. "The ee value of 4 was determined by HPLC analysis with chiral columns (Chiralcel OD-H for tc. Chiralpak AD-H for ta-b and $\mathbf{4 d - h )}$.

data and specific rotation with authentic samples. ${ }^{1+}$

In conclusion, we have developed a highly efficient catalytic asymmetric Michael reaction of fluoromalonates 2 to nitroalkenes 3 using air- and moisture-stable chiral nickel cataly st 1a. The desired $\gamma-n i t r o-\alpha$-fluoro carbonyl compounds $t$ were obtained in good to high yields and excellent enantioselectivities $\left(90-97 \%\right.$ ee) were observed. ${ }^{1+}$ We believe that this method provides an efficient route for the preparation of chiral $\gamma$-nitro- $\alpha$-fluorocarboxylic acid derivatives. and the availability of these compounds should facilitate medicinal chemical studies in various fields. Further details and application of this Michael addition of fluoromalonates will be presented in due course.

\section{References and Notes}

1. (a) Chambers, R. D. Firorine in Organic Chenistm; Blackwell: Oxford. 2004. (b) Kirsch, P. Hodem Flrorooryanic Chentstry: Swthesis, Reactivit, Applications: Wiley-VCH: Weinhem. 2004. For reviews: (c) Kirk. K. L. T. J. Flnorine Chem. 2006. 127, 1013. (d) Isanobor C.: OHagan. D. J. Fhonne (hem 2006, 127, 303. (e) Muller, K.: Faeh, C.: Diederich, F. Science 2007. 317,1881 . (f) Kirk. K. L. Org. Process Res. Dev. 2008, 12, 305

2. (a) Bolum, H.-T; Banner, D ; Bendels, S ; Kansy, M:: Kulur, B.; Muller, K.; Obstsander, U.; Stall, M. ChemBioChem 2004. S. 637. (b) Smart, B. R. T. J. Fhorine (hem. 2001, 109, 3. (c) Ismail, F. M. D. T. J. Fluorine Chem. 2002, 118, 27

3. Asymmetric Fluoroorganic Chentisty: Symthesis, Application, and Fuffre Directions, Ramachandran, P. V.. Ed.: ACS Symposium Series 746 American Chemical Society: Washington, DC, 2000.

4. (a) Leonard, J. Contentp. Org. Sinth. 1994, I, 387. (b) Perlmutter, $\mathrm{P}$. Conjugate Addition Reactions in Organic Sinthe sis: Pergamon: Oxford, 1992.

5. For recent reviews of asymmetric Michael addition reactions, see: (a) Krause, N.: Hoffinann-Reder, A. Shthesis 2001, 171. (b) Bener. O. M: Tedeschi. L.: Ender. D. Eur. J. Org. Chem. 2002. 1877 (c) Christoffers J: Baro. A. Angew Chem hit Ed. $2003,+2,1688$.

6. For recent reviews of organocatalytic asymmetric Michael addition, see: (a) Tsogoeva, S. B. Eur. J. Org. Chem. 2007, 1701. (b) Almasi, D.; Alonso, D. A.; Najera D. Tetrahedron: Armmem 2007, 18, 299.

7. (a) Ono, N. The Nino Group in Onganic Snthesis, Wiley-VCH: New York, 2001. (b) Calderan, G.; Seebach, D. Heh. Chim. Acta 1985, 68, 1592. (c) Ballini. R.: Petrini. M. Tetrahedron 2004, 60, 1017. (d) Czekelius, E.; Cameira, M. Angew. Chem. Iht. Ed. 2005, 4,612 .

8. For selected recent examples of Michael reactions of $1,3-$ dicarbonyl compounds, see: (a) Evans, D. A.; Seider, D. J. Am. Chem. Soc 2005, 127, 9958. (b) Terada, M.; Ube, H.; Yaguchi, Y.J.Am. Chem. Soc. 2006, 128, 14545. (c) Wu, F : Li, H.; Hong, R.; Deng, L. Angew. Chem., Int. Ed. 2006, 45, 947. (d) Wang, C.-T.; Zhang, Z.-H.; Dong, X.-Q.; Wu, X.-T. Chem. Conmmin. 2008, 1431 . (e) McGarraugh, P. G.; Brenner, S. E. Tetrahedron 2009, 65, 449. (f) Tan, B.; Zhang, X.; Chua, P. J.; Zhong, G. Chem Commun 2009,779

9. Asymmetric Michael-type reaction of fluoromalonates, see: (a) Kim, D. Y.; Kim, S. M.: Koh, K. O.: Mang, I. Y.; Lee, K. Buhl. Korean Chem. Soc. 2003, 24, 1425 . (b) Nichols, P. J.: DeMattei, I. A.: Barnett, B. R.: LeFur, N. A.: Chuans, T.-H.: Piscopio, A. D.; Koch, K. Org. Lett. 2006, 8, 1495

10. (a) Kim. D. Y.: Park. E. I. Org Letr. 2002 4. 545. (b) Park. E. I.: Kim, M. H.: Kim, D. Y. J. Org. Chem. 2004, 69, 6897, (c) Kim. S. M.: Kim. H. R.: Kim, D. Y. Org. Lett. 2005, 7, 2309 (d) Kim. H. R.; Kim, D. Y. Tetwhedron Lett. 2005, t6, 3115 . (e) Kang, Y. K.: Kim, D. Y. Tetrahedron Letr. 2006, 17, 4265. (f) Kim, S. M.; Kang, Y. K.; Lee, K.; Mang, J. Y.; Kim, D. Y. Bull Konean Chem. Soc. 2006, 47, 423. (g) Kang, Y. K.; Cho, M. T.; Kim, S. M.; Kim, D. Y. Synlett 2007, 1135 , (h) Cho, M. J.; Kang. Y. K: Lee, N. R.: Kim, D. Y. Bull. Korean Chem. Soc 2007, 28, 2191. (i) Kim, S. M.; Kang, Y. K.; Cho, M. J.; Mang, J. Y.; Kim, D. Y. Bull Korem Chem. Soc 2007, 28, 2435. (j) Lee, J. H, Bang, H. T.; Kim, D. Y. Sinlett 2008, 1821, (k) Kim, S. M. Lee, I. H.; Kim, D. Y. Sinlett 2008, 2659. (1) Jung. S. H.; Kim, D. Y. Tetrahedron Lett. 2008, 49,5527. (m) Mang. I. Y.; Kim. D. Y. Bull. Korean Chem. Soc. 2008, 29, 2091 (n) Kang. Y. K.; Kim, D. Y. Bull. Korean Chem. Soc. 2008, 29, 2093 (o) Lee, N. R:; Kim, S. M.: Kim, D. Y. Bull Korean Chem. Soc. 2009, $30,829$.

11. (a) Kim. D. Y. Bull. Korean Chem. Soc. 2008, 29. 2036 (b) Mang, T. Y.: Kwon, D. G.: Kim, D. Y. J. Fhorine Chem. 2009, 130, 259. (c) Mang, T. Y.: Kwon, D. G.; Kim, D. Y. Bull Koreon Chem. Soc. $2009,30,249$

12. (a) Evans, D. A.; Mito, S.; Seidel, D. J. Am. Chem. Soc. 2007, 129, 11583. (b) Fossy, J. S.; Matsubara, R.; Kivogara, H.; Kobayashi, S. Horg. Chemt 2008, 77,781 .

13. General procedure for asymmetric conjugate addition of fluoromalonates to nitroalkenes: To a stirred misture of ethyl fluoromalonate $(2,64.1 \mathrm{mg}, 0.36 \mathrm{mmol})$ and chiral nickel catalyst 1 a $(24.2 \mathrm{mg}, 0.03 \mathrm{mmol})$ in toluene $(0.6 \mathrm{~mL})$ was added nitroalkene $3(0.3 \mathrm{mmol})$ at room temperature. After being stirred for $9-20 \mathrm{~h}$. the reaction mixture was concentrated. The residue was purified by column chromatography on silica gel (hexane/ethyl acetate = $5 / 1)$ to afford desired product 4. 4a: $[\mathrm{\alpha}]_{\mathrm{D}}^{23}=15.4\left(\mathrm{c}=1.8, \mathrm{CHCl} \mathrm{s}_{3}\right)$ : ${ }^{l} \mathrm{H} N M R(200 \mathrm{MHz}, \mathrm{CDCl}) 1.01(\mathrm{t}, J=6.9 \mathrm{~Hz}, 3 \mathrm{H}), 1.35(\mathrm{t}, j=$ $7.0 \mathrm{~Hz}, 3 \mathrm{H}), 3.93-4.18(\mathrm{~m}, 2 \mathrm{H}), 4.32-4.42(\mathrm{~m}, 2 \mathrm{H}), 4.57(\mathrm{ddd}, J=$ $30.3,9.5,4.8 \mathrm{~Hz}, \mathrm{lH}), 4.81(\mathrm{dd}, J=13.4,9.5 \mathrm{~Hz}, \mathrm{lH}), 4.93(\mathrm{dd}$, $J=13.4,4.8 \mathrm{~Hz}, 1 \mathrm{H}, 7.29-7.32(\mathrm{~m}, 5 \mathrm{H}) \cdot{ }^{13} \mathrm{C}$ NMR $(50 \mathrm{MHz}$, $\left.\mathrm{CDCl}_{3}\right) 13.6,13.9,47.3(\mathrm{~d}, J=18.3 \mathrm{~Hz}), 62.9,63.6,75.5(\mathrm{~d}, J=$ $5.8 \mathrm{~Hz}, 94.5(\mathrm{~d}, J=200.2 \mathrm{~Hz}), 128.8,129.0,129.2,132.9,163.6$ $(\mathrm{d}, J=26.1 \mathrm{~Hz}), 164.6(\mathrm{~d}, J=25.5 \mathrm{~Hz}) \mathrm{t}_{\mathrm{R}} \mathrm{HPLC}(80.20, n$-hexane: $i-\mathrm{PrOH}, 220 \mathrm{~mm}, 1.0 \mathrm{~mL} / \mathrm{min}$ ) Chiralpak $\mathrm{AD}-\mathrm{H}, \mathrm{t}_{\mathrm{R}}=5.4 \mathrm{~min}$ (minor), $6.1 \mathrm{~min}$ (major). $97 \%$ ee.

14. After submission of this manuscript, a report on the organocatalytic Michael addition reaction of fluoromalonate has been published, see: Li, H.; Zu, L.: Xie, H.; Wang, W. Symhesis 2009. 1525

15. Hashimoto, S:; Furukawa, I:; Kuroda, T. Tetrahedron Left. 1980. 21,2857 . 